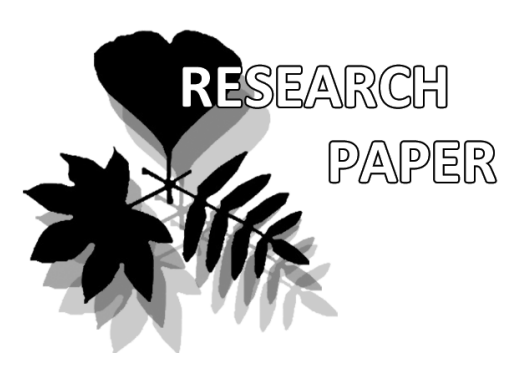

\title{
The steppe communities in urban ecosystems in the South Urals: syntaxonomy and conservations
}

\author{
Yaroslav M. Golovanov, Sergei M. Yamalov, \\ Larisa M. Abramova*
}

Yaroslav M. Golovanov

e-mail: jaro1986@mail.ru

Sergei M. Yamalov

e-mail: geobotanika@mail.ru

Larisa M. Abramova*

e-mail: abramova.lm@mail.ru

Botanical Garden-Institute of the Ufa Scientific Centre of the Russian Academy of Sciences, Ufa, 450080, Russia

* corresponding author

Manuscript received: 25.09 .2016

Review completed: 17.04.2017

Accepted for publication: 05.05.2017

Published online: 09.06.2017

\begin{abstract}
A B S T R A C T
The study of flora and vegetation of cities of the south of the Republic of Bashkortostan (Salavat, Ishimbai, and Meleuz) was performed during 2008-2012. Within the boundaries of cities, plots of surviving remnant steppe vegetation with a considerable concentration of rare plant species were found. For their characteristics, 90 relevés of steppe vegetation were performed. In the course of data analysis, we found that the syntaxonomy of the steppe vegetation of the cities of the Southern Industrial Zone of the Republic of Bashkortostan includes 1 class, 2 orders, 3 alliances, 7 associations, 2 subassociations, and 2 variants. In the coenoflora of plant communities of the class Festuco-Brometea recorded within the cities, of the 15 rare species listed in the Red Data Books of the Republic of Bashkortostan and the Russian Federation, 7 endemic, and 1 relic plant species were revealed. These species are unique for urban territories. We suggest creating 4 urban natural sanctuaries to protect steppe communities.
\end{abstract}

K e y w o r d s : South Urals, steppe vegetation, syntaxonomy, class Festuco-Brometea, rare species, conservation of vegetation cover

\section{P E 3 Ю M E}

Резюме

Голованов Я.М., Ямахов С.М., Абрамова И.М. Степные сообщества в урбанизированных экосистемах Южного Ураха: синтаксономия и вопросы охраны. Изучение флоры и растительности городов юга Республики Башкортостан (города Са^ават, Ишимбай и Мелеуз) проводились в течение 2008-2012 гг. В пределах границ городов были отмечены участки сохранившейся степной растительности с высокой концентрацией редких видов растений. Аля ее характеристики было выполнено 90 геоботанических описаний. В ходе их анализа было выяв ено, что синтаксономия степной растительности городов Южной промышленной зоны Республики Башкортостан вкцючает 1 класс, 2 порядка, 3 союза, 7 ассоциаций, 2 субассоциации и 2 варианта. В ценофлоре растительных сообществ класса Festuco-Brometea, отмеченных в пределах городов, выявлено 15 редких видов, занесенных в Красные книги Республики Башкортостан и Российской ФеАерации, 7 эндемичных и реликтовый вил растений. Аанные виды явАяются уникальными Аля городских территорий. А^я охраны степных сообществ предложено создать 4 городских памятника природы.

К $\boldsymbol{\Lambda}$ юч е в ы е с $\mathbf{\Lambda}$ в а : Южный Урал, степная растительность, синтаксономия, класс Festuco-Brometea, редкие, охрана растительного покрова
Cities are ecosystems in which a greater part of the planet's human population is concentrated. At the present time, the area of urbanized territories in the world occupies only $5 \%$ of the entire land, and about 3 billion people live in this area, from which it follows that in connection with an increase in the scale of urbanization, the study of vegetation cover of cities becomes increasingly urgent.

Within city boundaries, two main variants of vegetation communities can be distinguished. The first is anthropogenic. This includes purposefully created green plantings and very widespread communities of synanthropic species. The second variant represents natural ecotopes that pene- trated the administrative boundaries of the urban territory. In many cities, large fragments of natural vegetation: forest massifs, coastal phytocoenoses, swampy sites of river floodplains, inundated and dry meadows, steppe plots, etc. are retained. About two thirds of plants entering in the composition of urbanoflora are related to such refuges. Endemic, relic, and "Red Data Book" species are also rather frequently found on these territories.

All this indicates that it is necessary to include into the system of specially protected nature territories (SPNT) fragments of urbanized landscapes that are shelters of rare and protected species (Ilminskikh \& Tarasova 1992). 
They should be given the status of a nature reserve without allowing any commercial activity (Tikhomirov 1988). As Ilminskikh \& Porfirev (1979) noted, that according to the mode of use, an urban natural sanctuary belongs neither to protected areas, nor to nature reserves. Urban monuments can have a local importance since they can enter also in the local network of SPNT of a regional scale depending on the degree of preservation and uniqueness of the objects under protection present over their territory.

Taking into account the importance of this problem, it is necessary to note that the number of works concerning preservation of the flora of urban territories in the Russian Federation is rather small (Ilminskikh 1982, Chopik et al. 1986, Ilminskikh \& Tarasova 1992, Kuvaev et al. 1992, Dvinskikh et al. 2011, Buzmakov et al. 2012). However, during recent time, parallel to regional Red Data Books, Red Data Books of individual urban territories, as, for instance, "The Red Data Book of Nature of Saint Petersburg" (Noskov 2004) and "The Red Data Book of Moscow" (Samoilov \& Morozova 2011) have been established.

\section{MATERIAL AND METHODS}

We studied the flora and vegetation of cities of the Southern Industrial Zone of the Republic of Bashkortostan (Salavat, Ishimbai, and Meleuz) during 2008-2012 (Golovanov et al. 2011, 2014, Golovanov \& Abramova 2012 a, b, 2013). Some data on the key characteristics of the cities are presented in Table 1.
All three cities are located in the south of Cisurals of the Republic of Bashkortostan (RB) (Fig. 1) in the fluvial plain of the Belaya River, as well as its tributaries: in the city of Ishimbai - the Tairuk River and in the city of Meleuz - the Meleuz and Karan rivers.

The territory of the cities is characterized by a continental climate. The average temperature in January is $-14.7^{\circ} \mathrm{C}$, the average temperature of July $-+19.2^{\circ} \mathrm{C}$, and the average annual amount of precipitation is $410-460 \mathrm{~mm}$ (Yaparov 2005).

A large branch of the Kuibyshev railroad, as well as a federal highway Ufa-Orenburg pass over the territory of Salavat and Meleuz where the city of Ishimbai is slightly away from big transport routes. The cities also possess a developed industrial complex with maximum development in the city of Salavat - JSC Gazprom Neftekhim Salavat.

In the boundaries of these cities, plots of surviving steppe vegetation with a considerable concentration of rare plant species were revealed. For their characteristics, 90 relevés of steppe vegetation were performed. The size of the sample area depended on the size and uniformity of a community. Data were sampled according to the methods of Zürich-Montpellier school (Braun-Blanquet 1964, Westhoff \& Van Den Maarel 1978). Extended Braun-Blanquet 5-degree cover-abundance scale was used (Mirkin et al. 1989). All the relevés were imported into TURBOVEG, database program for storing of phytosociological data (Hennekens \& Schaminée 2001), and consecutively edited in the program MEGATAB (Hennekens 1995) and JUICE (Tíchý 2002).

Table 1. Characteristic of three cities of the Southern Industrial Zone of the Republic of Bashkortostan

\begin{tabular}{l|c|c|c|c|}
\hline City & $\begin{array}{c}\text { Date of } \\
\text { foundation }\end{array}$ & Area, $\mathbf{k m}^{2}$ & $\begin{array}{c}\text { Population, } \\
\text { thousands people }\end{array}$ & Natural zone \\
\hline Salavat & 1948 & 111.4 & 156.0 & Steppe \\
Ishimbai & 1940 & 103.0 & 68.1 & Forest-steppe \\
Meleuz & 1958 & 35.5 & 61.4 & Steppe \\
\hline
\end{tabular}

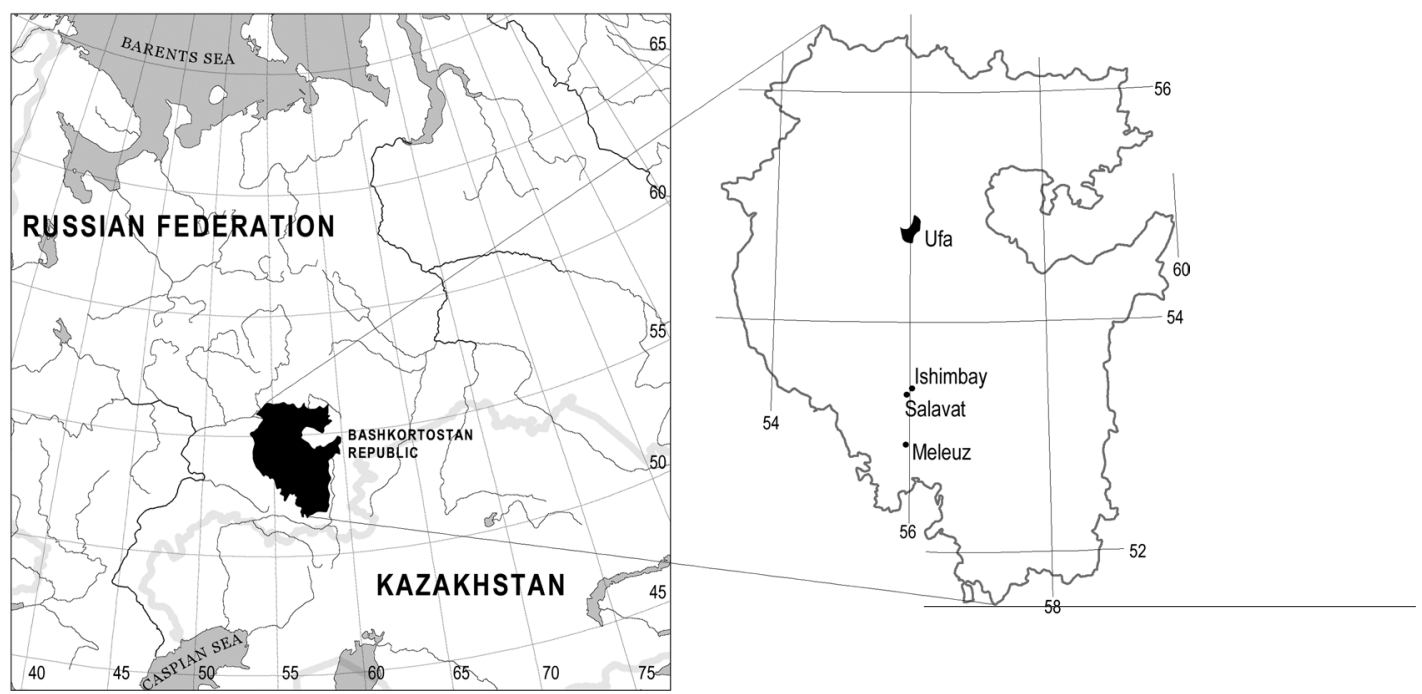

Figure 1 Localization of the cities on the Map of the Republic of Bashkortostan 
Table 2. Synoptic table of associations of the class FestucoBrometea

\begin{tabular}{|lccccccccc|}
\hline Syntaxon nr. & 1 & 2 & 3 & 4 & 5 & 6 & 7 & 8 & 9 \\
Mean nr. of species & 40 & 41 & 28 & 26 & 36 & 30 & 28 & 18 & 28 \\
Number of relevés & 14 & 6 & 12 & 6 & 10 & 5 & 10 & 12 & 10 \\
\hline
\end{tabular}

D.S. Ass. Poo angustifoliae-Stipetum pennatae

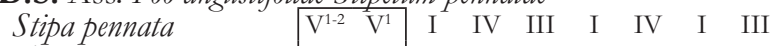
Amoria montana

\begin{tabular}{l|ccccccc} 
Thymus marschallianus & IV & V & III & . & III & III & I \\
Phleum phleoides & II & V & III & . & . & . & I
\end{tabular}

Onobrychis arenaria

III III

D.S. var. Helictotrichon desertorum

Helictotrichon desertorum $\mathrm{V}^{+-2}$. II I II . IV

D.S. var. Calamagrostis epigeios

Calamagrostis epigeios

D.S. Ass. Fragario viridis-Caraganetum fruticis

$\begin{array}{lllllllll}\text { Caragana frutex } & \mathrm{V} & \mathrm{V} & \mathrm{V}^{3-4} \mathrm{~V}^{3-4} & \mathrm{~V} & \mathrm{~V} & \text { III } & \text { III } & \mathrm{V}\end{array}$

D.S. Subass. F. v-C.f. festucetosum psendovinae

\begin{tabular}{lll|l|lllll} 
Festuca pseudovina & V & V & $V^{+-2}$ & IV & III & V & IV & V \\
Spiraea crenata & I & V & IV & IV & . & I &. & .
\end{tabular}

D.S. Subass. F. v.-C.f. poetosum angustifoliae and Ass. Poo angustifoliae-Stipetum pennatae

Poa angustifolia

Achillea millefolium

\begin{tabular}{|lll|l|l|l}
\hline III & V & V & $V^{1-2}$ & I \\
IV & V & III & IV \\
\hline
\end{tabular}

D.S. Ass. Astragalo austriacae-Stipetum pulcherrimae and Ass. Hedysaro grandiflori-Stipetum pulcherrimae

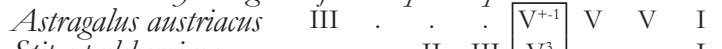

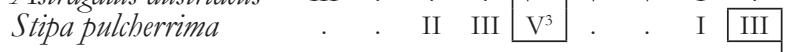

D.S. Ass. Scorzonero austriacae-Stipetum lessingianae

Stipa lessingiana

Scoryonera austriaca

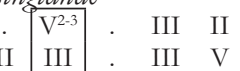

D.S. Ass. Salvio nutans-Stipetum korshinskyi

Stipa korshinskyi

$$
\text { II }
$$

\section{$\mathrm{V}^{2-3}$}

D.S. Ass. Trinio muricati-Centauretum sibiricae and

Ass. ассоциации Hedysaro grandiflori-Stipetum pulcherrimae

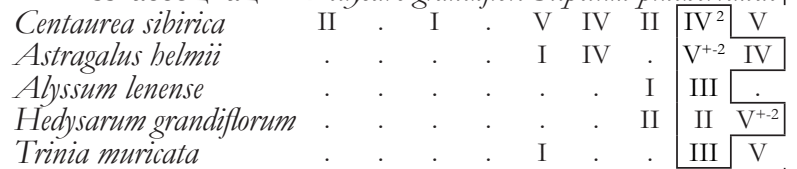

Trinia muricata

D.S. Ass. Hedysaro grandiflori-Stipetum pulcherrimae

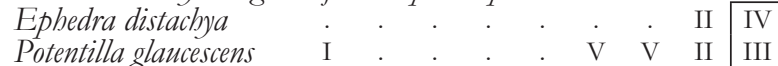
Jurinea ledebourii

D.S. Var. Astragalus belmii Artemisia salsoloides

D.S. Suball. Helictotricho desertori-Stipenion zalesskii

\begin{tabular}{|c|c|c|c|c|c|c|c|c|c|}
\hline arex pediformis & I & & 11 & & IV & $r$ & IV & 11 & $V$ \\
\hline Galium octonarium & III & I & III & I & $\mathrm{V}$ & III & $\mathrm{V}$ & III & $\mathrm{V}$ \\
\hline ypsophila altissima & III & & I & & $\mathrm{V}$ & II & IV & III & 2 \\
\hline Гanacetum kittaryanum & I & & & . & I & IV & III & III & 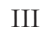 \\
\hline Polygala sibirica & I & & . & . & II & III & II & II & \\
\hline cabiosa isetensis & & & & & IV & $\mathrm{V}$ & III & III & V \\
\hline Echinops ruthenicus & & & & III & III & & II & IV & $v$ \\
\hline tuosum & I & . & & & & III & III & III & $\mathrm{V}$ \\
\hline a marshalliana & & & & & IV & II & II & IV & \\
\hline Gali & $\mathrm{V}$ & V & I & & & & I & & \\
\hline ne koriniana & II & & I & I & & II & . & I & \\
\hline atum & & & . & . & $\cdot$ & III & & $\mathrm{V}$ & IV \\
\hline ri & & & . & . & & IV & & III & III \\
\hline rophylla & & & . & . & & & III & III & $\mathrm{V}$ \\
\hline ierana & . & · & . & . & I & & & III & $\mathrm{V}$ \\
\hline & . & & . & 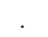 & & III & & II & \\
\hline & & & & & & & IV & III & \\
\hline & & & & & & & II & III & \\
\hline rshinskyi & & & & & & IV & & I & \\
\hline 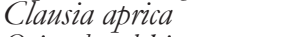 & & & & & & & & II & \\
\hline & & & & & & & & I & \\
\hline
\end{tabular}

Table 2. Continued

\begin{tabular}{|c|c|c|c|c|c|c|c|c|}
\hline Syn & 1 & 2 & 3 & 4 & 5 & 6 & 7 & 8 \\
\hline $\mathrm{Hec}$ & . & · & ${ }^{\circ}$ & r & . & . & . & II \\
\hline & $\cdot$ & . & & . & . & . & 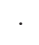 & I \\
\hline & 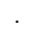 & . & & & . & . & & II \\
\hline
\end{tabular}

D.S. All. Helictotricho-Stipion and Ord. Helictotricho-Stipetalia

Artemisia austriaca III IV II . II III III III V

$\begin{array}{lllllllll}\text { Salvia stepposa } & \text { V } & \text { V } & \text { V } & \text { III } & \text { V } & \text { V } & \text { IV } & \text { I }\end{array}$

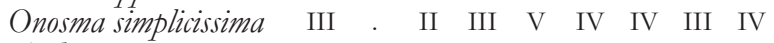

Androsace maxima I . . . . . II II

Euphorbia caesia II . . . . III IV III III V

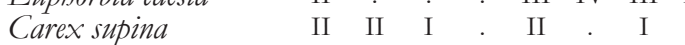

Galatella angustissima III . II . II . II

Potentilla bumifusa I . . . . III . II I

Achillea nobilis

Verbascum phoenicium II . .

D.S. All. Festucion valesiacae, Ord. Festucetalia valesiacae and Class Festuco-Brometea

$\begin{array}{llllllllll}\text { Stipa capillata } & \text { II } & \text { III } & \text { V } & \text { I } & \text { III } & \text { IV } & \text { II } & \text { II } & \text { III }\end{array}$

$\begin{array}{lllllllll}\text { Thalictrum minus } & \text { IV } & \text { V } & \text { V } & \text { III } & \text { IV } & \text { III } & \text { III } & \text { I }\end{array}$

$\begin{array}{lllllllll}\text { Galium verum } & \text { V } & \text { V } & \text { V } & \text { V } & \text { III } & \text { I } & \text { I } & \text { I }\end{array}$

$\begin{array}{llllllllll}\text { Veronica spicata } & \text { III } & \text { IV } & \text { I } & \text { II } & \text { I } & \text {. } & \text { I } & \text { I } & \text { I }\end{array}$

Fragaria viridis

Filipendula vulgaris

Medicago romanica

Cerasus fruticosa

Phlomoides tuberosa

Inula birta

Artemisia sericea

Plantago urvillei

Viola ambigua

Artemisia latifolia

Koeleria cristata

Campanula sibirica

Potentilla argentea

Aster amellus

Adonis vernalis

Dianthus versicolor

Festuca valesiaca

Anemone sylvestris

Seseli libanotis

Oxytropis pilosa

Trommsdorfia maculata

$\begin{array}{lllllll}\text { IV } & \mathrm{V} & \mathrm{V} & \mathrm{V} & \text { II } & \text { III } & \text { III }\end{array}$

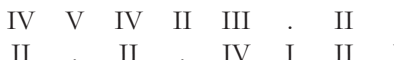

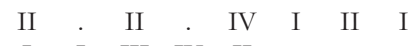

$\begin{array}{ccccccc}\text { I } & \text { I } & \text { III } & \text { IV } & \text { II } & \cdot & \text {. } \\ \text { IV } & \text { V } & \text { V } & \text { I } & \text { I } & \text {. } & \text { I }\end{array}$

$\begin{array}{lllllll}\mathrm{V} & \text { III } & \text { II } & \text { I } & \text { II } & \text {. } & \text { II }\end{array}$

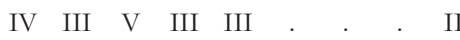

II IV I . II I III .

II . I . II I IV I

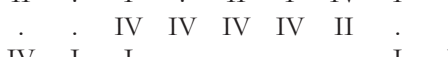

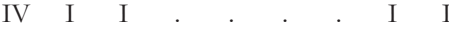

$\begin{array}{ccccccccc}\text { IV } & . & \text { II } & \text {. } & \text { I } & \text { V } & . & . & \text {. } \\ \text { I } & \text { V } & \text { II } & \text { I } & . & . & . & . & \text {. }\end{array}$

$\begin{array}{llll}\text { III } & \cdot & \cdot & \cdot \\ \text { III } & \cdot & \text { III } & \text { V }\end{array}$

II III III

. . . . I I V

D.S. Class Molinio-Arrhenatheretea

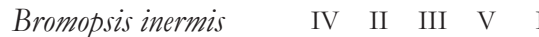

Astragalus danicus $\quad$ IV III I . . . I I

Elytrigia repens $\quad$ IV . III . II . III

Vicia cracca

Primula macrocalyx

Plantago media

Ranunculuspolyanthemos I III . II

Pimpinella saxifraga $\quad$ I $\quad$ II $\quad . \quad$. I

Carexpraecox . II . III .

Sanguisorba officinalis

D.S. Class Trifolio-Geranietea

Agrimonia asiatica

Origanum vulgare

Trifolium medium

Potentilla goldbachii

Pyrethrum corymbosum

Hypericum perforatum

Campanula bononiensis

D.S. Class Stellarietea mediae

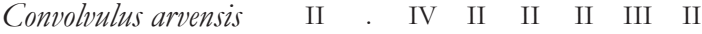

Lactuca serriola

Camelina microcarpa

D.S. Class Artemisietea vulgaris

$\begin{array}{llllllll}\text { Falcaria vulgaris } & \text { II } & \text { II } & \text { III } & \text { II } & \text { III } & \text { I } & \text { I }\end{array}$

Cichorium intybus

Cynoglossum officinale

Lappula squarrosa , 
Table 2. Continued

\begin{tabular}{|c|c|c|c|c|c|c|c|c|c|}
\hline Syntaxon nr. & 1 & 2 & 3 & 4 & 5 & 6 & 7 & 8 & 9 \\
\hline Euphorbia virgata & . & III & II & . & . & 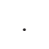 & . & ${ }^{\circ}$ & . \\
\hline \multicolumn{10}{|l|}{ Other species: } \\
\hline Galatella villosa & II & II & I & II & $\mathrm{I}$ & . & $\mathrm{I}$ & I & IV \\
\hline Thesium arvense & III & II & I & 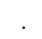 & I & . & II & I & I \\
\hline Scorzonera purpurea & III & III & III & . & II & . & II & I & . \\
\hline Eremogone longifolia & II & $\mathrm{V}$ & II & IV & . & . & II & . & . \\
\hline Asparagus officinalis & $\mathrm{I}$ & I & I & $\mathrm{V}$ & II & . & . & . & . \\
\hline Taraxacum proximum & II & II & I & ${ }^{\circ}$ & . & I & . & I & . \\
\hline Galium boreale & IV & IV & III & I & . & . & . & . & . \\
\hline Astragalus wolgensis & II & . & . & . & III & IV & $\mathrm{I}$ & I & $\mathrm{V}$ \\
\hline Vincetoxicum hirundinaria & III & I & . & III & II & . & . & . & . \\
\hline Dianthus andrajoinskianus & II & . & . & ${ }^{\circ}$ & I & . & I & I & . \\
\hline Otites wolgensis & III & I & 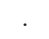 & $\cdot$ & I & . & . & . & I \\
\hline Carex caryopbyllea & IV & IV & I & ${ }^{\circ}$ & · & . & . & . & . \\
\hline Artemisia armeniaca & $\mathrm{V}$ & $\mathrm{V}$ & I & . & . & . & . & . & . \\
\hline Eryngium planum & I & I & . & IV & . & . & . & . & . \\
\hline Astragalus macropus & II & $\cdot$ & . & . & III & . & II & . & . \\
\hline Eremogone micradenia & II & 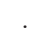 & II & . & 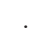 & . & II & . & . \\
\hline Campanula wolgensis & III & $\mathrm{V}$ & 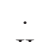 & I & . & . & . & 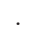 & . \\
\hline Artemisia marchalliana & $\mathrm{I}$ & . & II & . & 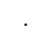 & . & . & 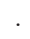 & III \\
\hline Inula aspera & II & 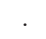 & 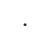 & . & I & I & . & . & . \\
\hline Silene chlorantha & II & $\cdot$ & . & ${ }^{\circ}$ & I & . & $\mathrm{I}$ & . & . \\
\hline Galatella biflora & $\mathrm{I}$ & $\cdot$ & . & IV & . & . & . & . & $\cdot$ \\
\hline Lupinaster albus & III & $\mathrm{V}$ & . & . & . & . & . & . & . \\
\hline
\end{tabular}

Table 2. Continued

\begin{tabular}{|c|c|c|c|c|c|c|c|c|c|}
\hline Syntaxon nr. & 1 & 2 & 3 & 4 & 5 & 6 & 7 & 8 & 9 \\
\hline Silaum silaus & I & . & . & IV & . & . & . & . & . \\
\hline Campanula wolgensis & . & . & III & I & . & . & r &. & . \\
\hline Eremogone sp. & . & . & . & 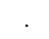 & II & III & . & . & . \\
\hline Xanthoselinum alsaticum & I & . & . & III & . & . & r & . & . \\
\hline Pilosella echioides & . & . & I & . & $\cdot$ & . & . & 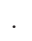 & III \\
\hline Artemisia pontica & $\mathrm{I}$ & . & e & II & . & . & . & . & . \\
\hline Melica transsilvanica & $\mathrm{I}$ & . & . & . & . & II & . & . & . \\
\hline Tragopogon dubius & . & . & r. & . & . & II & . & I & . \\
\hline Oxytropis spicata & II & . & . & . & I & . & . & . & . \\
\hline Centaurea ruthenica & $\mathrm{I}$ & $v^{\prime}$ & II & . & . & . & . & . & . \\
\hline Stachys recta & . & . & . & I & II & . & . & . & . \\
\hline Melampyrum arvense & . & $v^{\prime}$ & I & . & II & . & . & . & . \\
\hline Galium album & . & . & I & I & . & . & . & . & . \\
\hline Erucastrum armoracioides & I & . & . & . & I & . & . & . & . \\
\hline Goniolimon elatum & . & . & I & . & . & . & . & . & $\mathrm{I}$ \\
\hline Veronica prostrata & I & 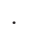 & . & . & 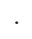 & . & I & $\cdot$ & . \\
\hline Vincetoxicum albowianium & . & 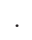 & . & . & I & . & I & $\cdot$ & . \\
\hline Alyssum turkestanicum & . & . & . & . & . & . & I & I & . \\
\hline Elytrigia lolioides & r & $\cdot$ & . & . & • & I & . & I & . \\
\hline Serratula gmelinii & . & · & I & I & . & . & . & . & . \\
\hline Senecio jacobaea & . & . & . & I & . & $\cdot$ & I & . & . \\
\hline Helictotrichon schellianum & $\mathrm{I}$ & I & r. & . & . & ${ }^{\circ}$ & . & . & . \\
\hline Hylotelephium stepposum & I & I & . & . & $\cdot$ & . & . & . & . \\
\hline
\end{tabular}

Taxa in one syntaxon: Aconogonon alpinum (2) +; Agrostis gigantea (4) r; Allium strictum (5) I; Androsace septentrionalis (2) I; Aster alpinus (7) II; Astragalus cicer (3) I; Astragalus sp. (8) r; Berteroa incana (3) II; Campanula persicifolia (2) r; Carduus acanthoides (1) r; C. nutans (1) r; Cephalaria uralensis (3) r; Crambe tataria (9) I; Echinops sphaerocephalus (1) I; Euphorbia semivillosa (4) r; Fritillaria ruthenica (2) r; Gagea pusilla (5) II; Galium aparine (3) I; Gentiana cruciata (1) r; Hieracium proceriforme (5) r; Knautia arvensis (3) r; Koeleria delavignei (2) r; Lactuca tatarica (8) I; Lathyrus pratensis (3) r; Lavatera thuringiaca (1) I; Linaria vulgaris (9) +; Linum uralense (9) r; Lonicera tatarica (3) I; Melampyrum argyrocomum (4) I; Melica altissima (3) I; Meniocus linifolius (8) I; Nepeta ucranica (3) r; Pedicularis kaufmannii (2) V; P. uralensis (1) r; Poa compressa (1) I; Polygala comosa (2) +; Potentilla recta (4) r; Primula macrocalyx (3) r; Ranunculus polyanthemos (2) r; Rhamnus cathartica (4) I; Rumex thyrsiflorus (1) r; Salvia verticillata (1) +; Serratula lycopifolia (1) r; Silene nutans (2) IV; Sisymbrium polymorphum (9) II; Solidago virgaurea (3) I; Sonchus arvensis 4 (II), Stachys officinalis (3) r; Stellaria graminea (4) IV; Steris viscaria (2) V; Stipa sareptana (7) r; Taraxacum proximum (4) r; Turritis glabra (2) II; Valeriana dubia (4) r; Veronica spuria (3) I; V. teucrium (3) r; Viola collina (3) + .

Syntaxon number: 1-2 Poo angustifoliae-Stipetum pennatae (1 Helictotrichon desertorum, 2 Calamagrostis epigeios), 3-4 Fragario viridisCaraganetum fruticis (3 F. v-C.f. festucetosum pseudovinae, 4 F. v.-C. f. poetosum angustifoliae), 5 Astragalo austriacae-Stipetum pulcherrimae, 6 Scorzonero austriacae-Stipetum lessingianae, 7 Salvio nutans-Stipetum korshinskyi, 8 Trinio muricati-Centauretum sibiricae, 9 Hedysaro grandiflori-Stipetum pulcherrimae.

\section{RESULTS AND DISCUSSION}

The hierarchical system of lower syntaxa of the urban steppe vegetation of South Urals includes 1 class, 2 orders, 3 alliances, 7 associations, 2 subassociations, and 2 variants.

\section{Prodromus of the urban steppe vegetation \\ Festuco-Brometea Br.-Bl. et Tx. ex Soó 1947}

Festucetalia valesiacae Br.-Bl. et Tx. ex Br.-Bl. 1949

Festucion valesiacae Klika 1931

Poo angustifoliae-Stipetum pennatae Yamalov et al. 2012

\section{Helictotrichon desertorum}

Calamagrostis epigeios

Amygdalion nanae V. Golub ex Yamalov 2011 Fragario viridis-Caraganetum fruticis Yamalov et Sultangareeva 2010

F. v.-C.f. festucetosum pseudovinae subass. nov. prov.

F. v.-C.f. poetosum angustifoliae subass. nov. prov.

\section{Helictotricho-Stipetalia Toman 1969}

Helictotricho desertori-Stipion rubentis Toman 1969 Astragalo austriacae-Stipetum pulcherrimae ass. nov. prov.

Scorzonero austriacae-Stipetum lessingianae ass. nov. prov.

Salvio nutantis-Stipetum korshinskyi ass. nov. prov.

Trinio muricati-Centauretum sibiricae Yamalov et al. 2011

Hedysaro grandiflori-Stipetum pulcherrimae ass. nov. prov.

\section{Description of syntaxa of the steppe vegetation}

Synoptic table of associations of class Festuco-Brometea is given below in table 2 .

\section{Association Poo angustifoliae-Stipetum pennatae} (Table 2, columns 1-2)

Diagnostic species (D.S.): Amoria montana, Centaurea scabiosa, Onobrychis arenaria, Phleum phleoides, Poa angustifolia, Stipa pennata, Thymus marschallianus. 
Associations of the community represent widespread meadow steppes where Stipa pennata represents a zonal type of steppes in the forest-steppe zone. Among herbaceous plants, species of the order Festucetalia valesiacae and the class Festuco-Brometea dominate. In the association composition, two variants Helictotrichon desertorum and Calamagrostis epigeios are distinguished. Coenoses of the variant Helictotrichon desertorum are characterized by a high constancy of $H$. desertorum, as well as by the presence of the block of species of the order Helictotricho-Stipetalia, unlike communities of the variant Calamagrostis epigeios with a more mesophytic floristic composition. The number of species per relevé ranges from 33-52 (average 40). Cover total $=$ $80-90 \%$ on sample plot $100 \mathrm{~m}^{2}$. Average height of herbs is $35-55 \mathrm{~cm}$. Association communities are confined to slopes of hills with different exposure with a relative inclination of 5 to $35^{\circ}$.

The communities of the association were recorded in the city of Ishimbai (Alebastrovaya Mountain, steppes near Peregonnyi and Termen-Elga settlements) and in the City of Salavat - steppe near OJSC Salavatsteklo.

\section{Variant Helictotrichon desertorum}

(Table 2, column 1)

D.S.: Helictotrichon desertorum. The number of species per relevé ranges from 33 to 52 (average 40). Cover totals $80-90 \%$ on sample plot $100 \mathrm{~m}^{2}$. Average height of herbs is $35-50 \mathrm{~cm}$. Communities of the variant are confined to slopes of different exposure with a relative inclination of $5-25^{\circ}$.

\section{Variant Calamagrostis epigeios}

(Table 2, column 2)

D.S.: Calamagrostis epigeios. The number of species per relevé ranges from 34 to 49 (average 41). Cover totals $85-90 \%$ on sample plot $100 \mathrm{~m}^{2}$. Average height of herbs is $45-55 \mathrm{~cm}$. Communities of the variant are confined to slopes of mainly northern exposure with a relative inclination of $20-35^{\circ}$.

\section{Association Fragario viridis-Caraganetum fruticis \\ (Table 2, columns 3-4)}

D.S.: Caragana frutex (dominant).

The association combines communities of steppe shrubs dominated by Caragana frutex that are the most widespread communities of shrub steppes of the foreststeppe zone of the South Urals (Mirkin 2010). Under the canopy of Caragana frutex with more mesophytic conditions contain different species of the order of meadow steppes Festucetalia valesiacae, as well as species of the class Molinio-Arrbenatheretea occur. Two subassociations are distinguished in the composition of the association: $\boldsymbol{F} \cdot \boldsymbol{v}_{\mathbf{v}} \boldsymbol{- C}$. $f$. festucetosum pseudovinae (develops under more xerophytic conditions) and $\boldsymbol{F} \cdot \boldsymbol{v}$ - $\boldsymbol{C}$. $f \cdot$ poetosum angustifoliae (typical for more mesophytic conditions). The number of species per relevé ranges from 24 to 34 (average 29). Cover totals $80-90 \%$ on sample plot $64 \mathrm{~m}^{2}$. Average height of shrubs is $60-150 \mathrm{~cm}$, herbs $-30-65 \mathrm{~cm}$.

The association communities were recorded in the city of Ishimbai (Alebastrovaya Mountain, steppes near Pregonnyi and Termen-Elga settlements), in the city of Salavat - steppe near OJSC Salavatsteklo, and in the city of Meleuz - steppe near OJSC Meleuz Mineral Fertilizers.

\section{Subassociation $F$. v.-C. f. festucetosum pseudovinae}

D.S.: Festuca pseudovina, Spiraea crenata.

The number of species per relevé ranges from 25 to 33 (average 20). Cover totals $80-90 \%$ on sample plot $64 \mathrm{~m}^{2}$. Average height of shrubs is $60-75 \mathrm{~cm}$, herbs $-30-55 \mathrm{~cm}$. The subassociation communities are confined to sunny slopes with a relative inclination of $5-10^{\circ}$.

\section{Subassociation $F$. v. $-C$. $f$. poetosum angustifoliae}

D.S.: Achillea millefolium, Poa angustifolia.

The number of species per relevé ranges from 27 to 32 (average 28). Cover totals $90 \%$ on sample plot $64 \mathrm{~m}^{2}$. Average height of shrubs is $60-70 \mathrm{~cm}$, herbs $-40-55 \mathrm{~cm}$. The subassociation communities are confined to slopes of mainly eastern exposure with a relative inclination of $5-15^{\circ}$.

\section{Association Astragalo austriacae-Stipetum pulcherrimae}

(Table 2, column 5)

D.S.: Astragalus austriacus, Stipa pulcherrima (dominant).

Communities are characterized by dominance of Stipa pulcherrima and represent a zonal type of steppes of Bashkirian Cisurals. In the herbage, species of the alliance Helictotricho-Stipion and order Helictotricho-Stipetalia dominate, and species of the suballiance of mountain steppes Helictotricho desertori-Stipenion rubentis Toman 1969 are also rather widely represented. The number of species per relevé ranges from 32 to 41 (average 36). Cover totals $80-90 \%$ on sample plot $64-100 \mathrm{~m}^{2}$. Average height of herbs is $35-45 \mathrm{~cm}$. The association communities are confined to sunny slopes of hills with mainly southern exposure and a relative inclination of 2 to $20^{\circ}$.

The association communities were recorded in the city of Ishimbai (Alebastrovaya Mountain and steppes near Perogonnyi and Termen-Elga settlements) and in the city of Salavat - steppes near SPNT Salavatsteklo.

Association Scorzonero austriacae-Stipetum lessingianae (Table 2, column 6)

D.S.: Scorzonera austriaca, Stipa lessingiana (dominant).

Communities are characterized by dominance of Stipa lessingiana. The association represents southern dry feathergrass steppes zonal type of steppes of the South Urals typical of the steppe zone. The floristic composition is formed by species of the alliance Helictotricho-Stipion and order Helictotricho-Stipetalia, species of the suballiance of mountain steppes Helictotricho desertori-Stipenion rubentis are also widely represented, which is explained by the location of communities at very sunny mountain slopes. The number of species per relevé ranges from 26 to 35 (average 30). Cover totals $60-80 \%$ on sample plot $64 \mathrm{~m}^{2}$. Average height of herbs is $35-45 \mathrm{~cm}$. The association communities are confined to slopes of hills with the southern exposure and a relative inclination of 10 to $30^{\circ}$.

The association communities were recorded only near the city of Ishimbai (steppes near Peregonnyi and TermenElga settlements).

Association Salvio nutanti-Stipetum korshinskyi (Table 2, column 7)

D.S.: Stipa korshinskyi (dominant) 

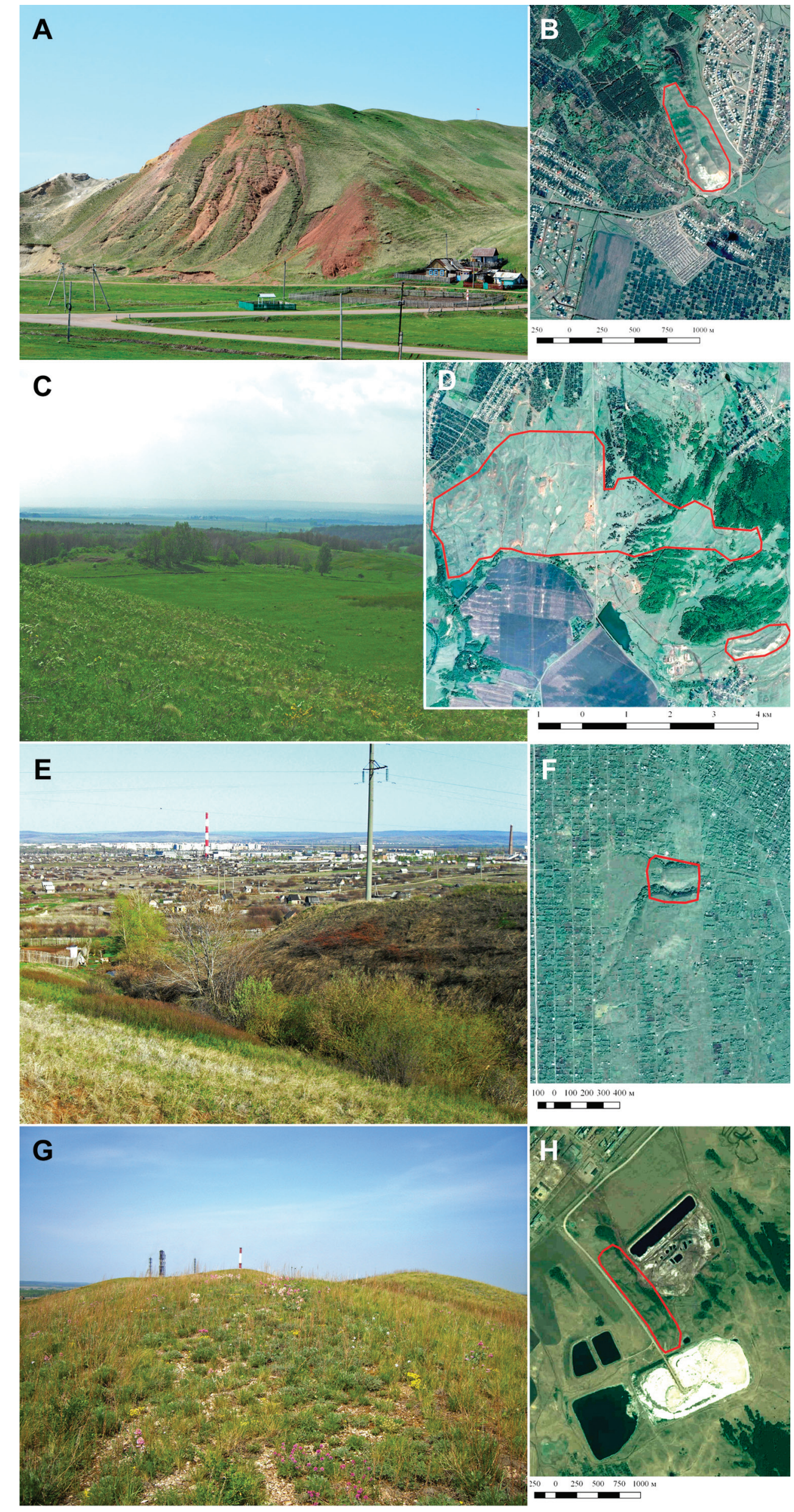

Figure 2 Appearances (left picture) and maps (right picture) of urban nature sanctuaries: A, B - Alebastrovaya Mountain; C, D - Steppes near Peregonnyi and Termen-Elga settlements; E, F - Steppes near SPNT Salavatsteklo; G, $\mathrm{H}$ - Steppes near SPNT Meleuz Mineral Fertilizers 
Communities are characterized by domination of Stipa korshinskyi. The number of species per relevé ranges from 20 to 38 (average 28). Cover totals 55-80\% on sample plot $64-100 \mathrm{~m}^{2}$. Average height of herbs is $35-45 \mathrm{~cm}$. The association communities are confined to sunny slopes of hills with different, mainly southwestern and southeastern exposure with a relative inclination of 20 to $40^{\circ}$.

The association communities were localized only in the city of Ishimbai (Alebastrovaya Mountain, steppes near Peregonnyi and Termen-Elga settlements).

\section{Association Trinio muricati-Centauretum sibiricae} (Table 2, column 8)

D.S.: Alyssum lenense, Astragalus helmii, Centaurea sibirica, Hedysarum grandiflorum, Trinia muricata.

The association combines communities of petrophytic steppes first described on Tratau and Yuraktau mountains (Yamalov et al. 2011). The communities recorded within the cities of Ishimbai and Meleuz extend the range of this association. However, compared to coenoses recorded by Yamalov et al. (2011), the association communities described by us are floristically poorer, which can be related to the anthropogenic impact on these habitats. The number of species per relevé ranges from 13 to 28 (average 21). Cover totals $25-80 \%$ on sample plot $20-64 \mathrm{~m}^{2}$. Average height of herbs is $15-35 \mathrm{~cm}$. The association communities are confined to strongly insolated mountain slopes of hills of mainly southern exposure with a relative inclination of 0 to $50^{\circ}$.

The association communities were recorded in the city of Ishimbai (Alebastrovaya Mountain and steppes near Peregonnyi and Termen-Elga settlements) and in the city of Meleuz - steppes near SPNT Meleuz Mineral Fertilizers. Association Hedysaro grandiflori-Stipetum pulcherrimae (Table 2, column 9)

D.S.: Ephedra distachya, Hedysarum grandiflorum, Jurinea ledebourii, Potentilla glaucescens, Stipa pulcherrima.

The association combines communities of petrophytic steppes of Bashkirian Cisurals. These communities belong to a specific variant Astragalus helmii (D.S.: Astragalus helmii, Artemisia salsoloides) typical of the southern part of Bashkirian Cisurals. The number of species per relevé ranges from 27 to 33 (average 30). Cover totals $65-70 \%$ on sample plot $64 \mathrm{~m}^{2}$. Average height of herbs is $22-35 \mathrm{~cm}$. The association communities are confined to hill slopes with mainly eastern exposure and a relative inclination of 10 to $20^{\circ}$.

The association communities were recorded only in the city of Meleuz (steppes near SPNT Meleuz Mineral Fertilizers).

\section{Problems of preservation of vegetation of urban natural sanctuaries}

In the coenoflora of plant communities of the class Festuco-Brometea, recorded within the limits of cities, a number of rare, relic, and endemic plant species was recorded. These species are unique for urban territories and occur only at the given sites within urban boundaries with relatively undisturbed steppe landscapes. Most rare plants are contained in petrophytic-steppe (Artemisia salsoloides, Asperula petraea, Astragalus helmii, Carex pediformis, Ephedra di- stachya, Hedysarum grandiflorum, Koeleria sclerophylla, etc.) and steppe (Oxytropis spicata, Stipa lessingiana, S. pennata, S. pulcherrima etc.) species.

The phytosociological attribution of endemic, relic, and rare species in the vegetation cover of urban natural sanctuaries is shown in Table 3.

It is seen from the table that the greatest number of rare species is concentrated in steppe communities of associations Trinio muricati-Centauretum sibiricae and Hedysaro grandiflori-Stipetum pulcherrimae recorded on the territory of the Alebastrovaya Mountain, steppes near Peregonnyi and Termen-Elga settlements, and steppes near SPNT Meleuz Mineral Fertilizers. Communities of the association Astragalo austriacae-Stipetum pulcherrimae spread over the territory of the Alebastrovaya Mountain, steppes near Peregonnyi and Termen-Elga settlements while steppes near SPNT Salavatsteklo have also a high nature-protective value.

\section{CONCLUSIONS}

For conservation of rare types of communities we suggest the establishment of urban natural sanctuaries:

1. "Alebastrovaya Mountain" (city of Ishimbai) (Figs. 2-3) represents an elevated watershed with minimal relative heights up to $234 \mathrm{~m}$ located along the right bank of the Tairuk River. Vegetation comprises mainly different variants of steppes (from meadow to mountain), plantings of pine are located to the north on the mountain. The mountain vegetation is subject to strong recreational impact. The area of the urban natural sanctuary is $0.16 \mathrm{Km}^{2}$.

2. "Steppes near Peregonnyi and Termen-Elga settlements" (city of Ishimbai) (Figs. 4-5). The western part of the locality is occupied by meadow and true steppes. A considerable part of hills in the eastern part is covered by broad-leaved oak forests, as well as by different steppe variants. The vegetation of the hills is vulnerable to a strong negative impact from livestock grazing and heavier than normal recreation. The area of the urban natural sanctuary is $4.53 \mathrm{~km}^{2}$.

3. "Steppes near SPNT Salavatsteklo" (city of Salavat) (Figs. 6-7). The vegetation of these habitats is represented by surviving remnants of different variants of steppes at sites inaccessible for commercial activity (slopes of gullies). The area of the urban natural sanctuary is $0.03 \mathrm{~km}^{2}$.

4. "Steppes near SPNT Meleuz Mineral Fertilizers" (city of Meleuz) (Figs. 8-9). Flora and vegetation of this elevated watershed being surrounded by technogenic ecotopes is unique for the city of Meleuz. Vegetation of hills includes mainly different steppe variants, as well as small separate oak stands. The vegetation of slopes is subject to a strong anthropogenic effect due to close proximity to industrial sites. The area of the urban natural sanctuary is $0.11 \mathrm{~km}^{2}$.

\section{LITERATURE CITED}

Buzmakov, S.A., G.A. Voronov, S.A. Kulakova et al. 2012. Specially protected nature territories of the City of Perm. Perm State University, Perm, 204 pp. (in Russian) [Бузмаков С.А., Воронов Г.А., Кулакова С.А. и Ар. 2012. Особо охраняемые территории города Перми. Пермский государственный университет: Пермь, 204 с.]. 
Chopik, V.N., A.N. Krasnova \& A.I. Kuzmichev 1986. Model wild-growing flora of urbanized territories Lysaya gora natural boundary. Botanicheskii Zhurnal 71(8): 11361141 (in Russian) [Чопик В.Н., Краснова А.Н., Кузьмичев А.И. 1986. Эталон естественной флоры урбанизированных территорий - урочище Аысая Гора / / Ботанический журнаА. Т. 71, № 8. С. 1136-1141].

Dvinskikh, S.A., N.G. Maksimovich, K.N. Maleev \& O.V. Larchenko 2011. Ecology of the Forest-Park Zone of the City. Nauka, Saint Petersburg, 154 pp. (in Russian) [Авинских С.А., Максимович Н.Г., Малеев К.Н., Аарченко О.В. 2011. Экология месопарковой зоны города. Санкт-Петербург: Наука, 154 с.].

Ilminskikh, N.G. \& V.S. Porfirev 1979. On the problem of nature conservation on urban territory. In: Issues of plant biology and conservation of vegetation world. Mezhvuzovskii sbornik nauchnykh trudov, Ivanovo, pp. 48-54 (in Russian) [ИАьминских Н.Г., Порфирьев В.С. 1979. К проб́леме охраны природы на городской территории // Вопросы биологии растений и охраны растительного мира. Межвузовский сборник научных трудов. Иваново. С. 48-54].

Il'minskikh, N.G. \& E.M. Tarasova 1992. On the conservation of phytogenofond of urbanized flora. Vestnik Udmurtskogo Universiteta 3:90-93. (in Russian) [ИАьминских Н.Г., Тарасова Е.М. 1992. К охране фитогенофонда урбанизированной флоры // Вестник $\mathrm{Y}_{\mathrm{A}}$ муртского университета. № 3. С. 90-93].

Golovanov, Ya.M., S.S. Petrov \& L.M. Abramova 2011. Vegetation of the city of Salavat (Republic of Baskortostan). I. Higher Aquatic Vegetation (classes Lemnetea and Potametea). Rastitel'nost' Rossii 19:55-71 (in Russian) Голованов Я.М., Петров С.С., Абрамова А.М. 2011. Растительность города Салавата (Респуб̆лика Башкортостан). I. Высшая водная растительность (классы Lemnetea и Potametea) // Растительность России. № 19. С. 55-71]

Golovanov, Ya.M. \& L.M. Abramova 2012a. Vegetation of the city of Salavat (Republic of Bashkortostan). II. Coastal aquatic vegetation (classes Phragmito-Magnocaricetea and Isoëto-Nanojuncetea). Rastitel'nost' Rossii 20: 3-26 (in Russian) [Голованов Я.М., Абрамова А.M. 2012а. Растительность города Салавата (Республика Башкортостан). II. Прибрежно-водная растительность (классы Phragmito-Magnocaricetea и Isö̈to-Nanojuncetea) // Растительность России. № 20. С. 3-26].

Golovanov, Ya.M. \& L.M. Abramova 2012b. Vegetation of the city of Salavat (Republic of Bashkortostan). III. Synanthropic vegetation (classes Bidentetea tripartitae, Stellarietea mediae, and Artemisietea vulgaris). Rastitel'nost' Rossii 21: 34-65 (in Russian) ГГолованов Я.М., Абрамова А.M. 2012б. Растительность города Салавата (Республика Башкортостан). III. Синантропная растительность (классы Bidentetea tripartitae, Stellarietea mediae и Artemisietea vulgaris) // Растительность России. № 21. С. 34-65].

Golovanov, Ya.M. \& L.M. Abramova 2013. Vegetation of the city of Salavat (Republic of Bashkortsan). IV. Synanthropic vegetation (classes Polygono arenastri-Poëtea annuae, Galio-Urticetea, and Robinietea). Rastitel'nost' Rossii 22: 11-20 (in Russian) [Голованов Я.М., Абрамова А.M. 2013. Растительность города Салавата (Респуб̆лика Башкортостан). IV. Синантропная растительность (кょассы Polygono arenastri-Poёtea annuae, Galio-Urticetea и Robinietea) // Растительность России. № 22. С. 11-20].

Golovanov, Ya.M., S.M. Yamalov \& L.M. Abramova 2014. Vegetation of the city of Salavat (Republic of Bashkortostan). V. Natural and semi-natural herbaceous vegetation (classes Molinio-Arrhenatheretea, Festuco-Brometea, and The-
ro-Salicornietea). Rastitel'nost' Rossii 24: 13-37 (in Russian) [Голованов Я.М., Ямалов С.М., Абрамова А.М. 2014. Растительность города Салавата (Респуб̆лика Башкортостан). V. Естественная и полуестественная травяная растительность (Классы Molinio-Arrhenatheretea, FestucoBrometea и Thero-Salicornietea) / / Растительность России. № 24. C. 13-37].

Gorchakovskii, P.L. 1969. Main problems of historic phytogeography of the Urals. In: Trudy Instituta Ekologii Rastenii I Zhivotnykh. Ural Branch of the Academy of Sciences of the USSR, Sverdlovsk, 207 pp. (in Russian) [Горчаковский П.А. 1969. Основные проблемы исторической фитогеографии Ура^а // Труды института экологии растений и животных. СверАловск: Уральское отделение Академии наук СССР, 207 с.].

Kulikov, P.V. 2005. Synopsis of flora of Chelyabinsk oblast (vascular plants). Geotur, Yekaterinburg, 537 pp. (in Russian) [Куликов П.В. 2005. Конспект флоры Челябинской области. Екатеринбург: Геотур. 537 с.].

Kuvaev, V.B., M.L. Shelgunova \& L.K. Konstantinov 1992. Flora of Znamenskoe and its vicinity. Nauka, Moscow, 356 pp. (in Russian) [Куваев В.Б., Шелгунова М.А., Константинов А.К. 1992. ФАора окрестностей Знаменского. Москва: Наука. 356 с.].

Mirkin, B.M. (ed.) 2010. Flora and vegetation of the National Park "Bashkiria" (syntaxonomy, anthropogenic dynamics, and ecological zonation). Academy of Sciences of the Republic of Bashkortostan, Gilem, Ufa, 512 pp. (in Russian) [ФАОра и растительность Национального парка Башкирия (синтаксономия, антропогенная Аинамика и экологическое зонирование) / под реА. Б.М. Миркина. Уфа: Акалемия наук Республики Башкортостан, Гилем, 2010. 512 c.].

Mirkin, B.M. (ed.) 2011. The Red Data Book of the Republic of Bashkortostan, Vol. 1: Plants and Fungi. Media-Print, Ufa, 384 pp. (in Russian) [Красная Книга Респуболики Башкортостан / под ред. Б.М. Миркина. Уфа: МедиаПринт, 2011. 384 с.].

Mirkin, B.M., G.S. Rozenberg \& L.G. Naumova 1989. Dictionary of concepts and terms of modern phytocoenology. Nauka, Moscow, 222 pp. (in Russian) [Миркин Б.M., Розенберг Г.С., Наумова А.Г. 1989. Словарь понятий и терминов современной фитоценологии. Москва: Наука. 222 с.].

Noskov, G.A. (ed.) 2004. The Red Data Book of nature of Saint Petersburg. Saint Petersburg: Profession. 414 pp. (in Russian) [Красная Книга природы Санкт-Петербурга / поА ред. Г.А. Носкова. Санкт-Петербург: Профессион. 414 c.].

Samoilov, B.L. \& G.V. Morozova (eds.) 2011. The Red Data Book of Moscow. Department of Nature Use and Environmental Conservation of Moscow, Moscow, 930 pp. (in Russian) [Красная Книга города Москвы / под ред. Б. $\Lambda$. Самойлова и Г.В. Морозовой. Москва: Аепартамент природопользования и охраны окружающей среды города Москвы, 2011. 930 c.].

Tikhomirov, V.N. 1988. On the Volume of Concept "Nature Sanctuaries". In: Urgent Issues of Botany in the USSR. Nauka, Alma-Ata, 443 pp. (in Russian) [Tихомиров B.H. 1988. О проблеме понятия «памятники природы» // Актуальные вопросы ботаники в СССР. А^ма-Ата: Наука. 443 с.].

Trutnev, Yu.P. et al. (eds.) 2008. The Red Data Book of the Russian Federation (Plants and Fungi). KMK Press, Moscow, 855 pp. (in Russian) [Красная Книга Российской Федерации. Т. 1. Растения и грибы / под редакцией Ю.П. Трутнева и Ар. Москва: КМК. 855 с.].

Yamalov, S.M., A.V. Bayanov, V.B. Martynenko, A.A. Mulda- 
shev \& P.S. Shirokhikh 2011. Endemic associations of petrophytic steppes of paleoreefs of the South Urals, Rastitel'nost' Rossii 19: 117-127 (in Russian) [Ямалов C.M., Баянов А.В., Мартыненко В.Б., Мулдашев А.А., Широких П.С. 2011. Эндемичные ассоциации петрофитных степей палеорифов Южного Урала // Растительность России. № 19. С. 117-126].
Yaparov, I.M. (ed.) 2005. Atlas of the Republic of Bashkortostan. Kitap, Ufa, 420 pp. (in Russian) [Атлас Республики Башкортостан / гл. реА. И.М. Япаров. Уфа: Китап, 2005. 420 с.]. 